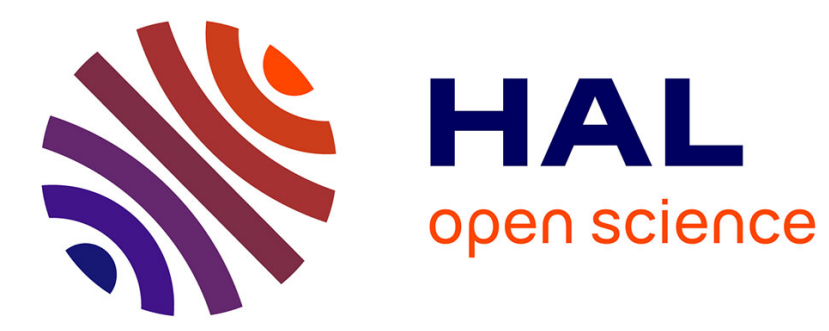

\title{
Une analyse compositionnelle de Quoi que ce soit comme universel
}

\author{
Francis Corblin
}

\section{To cite this version:}

Francis Corblin. Une analyse compositionnelle de Quoi que ce soit comme universel. Langue française, 2010, 2 (166), pp.17-50. ijn_00540301

\section{HAL Id: ijn_00540301 https://hal.science/ijn_00540301}

Submitted on 26 Nov 2010

HAL is a multi-disciplinary open access archive for the deposit and dissemination of scientific research documents, whether they are published or not. The documents may come from teaching and research institutions in France or abroad, or from public or private research centers.
L'archive ouverte pluridisciplinaire HAL, est destinée au dépôt et à la diffusion de documents scientifiques de niveau recherche, publiés ou non, émanant des établissements d'enseignement et de recherche français ou étrangers, des laboratoires publics ou privés. 
Une analyse compositionnelle de "Quoi que ce soit" comme universel*

Francis Corblin

Université Paris-Sorbonne

\& Institut Jean Nicod (CNRS-ENS-EHESS)

Le présent article étudie les formes quoi (qui, où) que ce soit en se situant à l'intersection de deux points de vue :

1) un point de vue "distributionnel" au sens large, qui considère ces formes comme des items lexicaux et s'attache à en déterminer les contraintes d'emploi et la sémantique.

$C^{\prime}$ est ce point de vue qui conduit à traiter ces expressions complexes comme des items de choix libre. Il est adopté notamment dans Vlachou (2007), et fournit une cartographie très fine de leurs contraintes d'emploi et de leurs valeurs sémantiques.

2) un point de vue "compositionnel", entrant dans la structure interne de ces expressions, qui les voit comme des structures analysables, des syntagmes composés d'éléments que l'on retrouve dans d'autres structures, et qui ont leur propre sémantique. Dans cette direction, on rencontre la notion de concession, ou d'opposition, utilisée dans les grammaires pour quoi que ce soit.

Dans cet article, on voudrait tenter d'établir un lien entre ces deux directions de recherche. Plus précisément, on voudrait déterminer s'il est possible d'expliquer que quoi que ce soit ait un comportement qui l'apparente aux termes de choix libre, en partant des items qui le composent, et qui le rangent du côté de la concession.

La thèse que nous allons défendre est que quoi que ce soit est un quantificateur universel associé à deux propriétés spécifiques: il implique un élargissement maximal de son domaine de quantification, et il prend portée maximale sur la phrase où il se trouve.

Nous soutiendrons que cette analyse s'applique à tous les emplois de la forme, parfois scindés en emplois de choix libre et emplois comme terme de polarité 1 .

Nous essaierons de montrer que cette sémantique dérive directement de l'interprétation de la construction (quoi que ce soit + relative) comme complétive disjonctive à interprétation conditionnelle, structure illustrée par (1) :

(1) Que Pierre ou Marie vienne, partez immédiatement.

Par rapport à la proposition de Vlachou (2007), qui traite quoi que ce soit comme un indéfini d'élargissement, le présent travail est une tentative pour montrer qu'il est possible de proposer une version de cette même approche reposant sur une analyse de la forme comme quantificateur universel à portée large, la notion d'élargissement restant un point commun des deux approches.

En ce qui concerne les relations entre quoi que ce soit et any, il y a de telles différences de distribution entre les deux formes ${ }^{2}$, malgré des recouvrements partiels évidents, que l'analyse

\footnotetext{
La rédaction de cet article a été facilitée par une délégation CNRS à l'institut Jean Nicod. L'article a beaucoup bénéficié de la relecture critique de Evangelia Vlachou. Je la remercie sincèrement de toutes ses observations, sur la forme et le fond. Je remercie également un des relecteurs de la revue pour ses précieuses observations, notamment en ce qui concerne les données. Les erreurs qui subsistent sont naturellement les miennes.

Voir Muller (2006) pour une illustration de la différence et l'expression de quelques doutes sur la possibilité de séparer strictement ces emplois.
} 
de l'une de ces formes ne permet aucune conclusion en ce qui concerne l'autre. La littérature sur any fournit cependant de précieux éléments d'analyse dans la mesure où plusieurs hypothèses ont été avancées, discutées et parfois réfutées. Il est donc souvent très intéressant de vérifier si les objections sont applicables au traitement que l'on propose pour la forme française.

\section{Quoi que ce soit et son paradigme : tableau des données.}

Quoi que ce soit est traité dans Vlachou (2007, p.90), comme un membre du paradigme morphologique des termes de choix libre concessifs. Dans ce large ensemble, on peut retenir comme paradigme étroit de quoi que ce soit uniquement les segments qui admettent la distribution d'un groupe nominal ou d'un GN prépositionnel argument du verbe principal. La liste est donnée sous (2) et illustrée sous (3-6) :

(2) Qui que (ce soit), Quoi que (ce soit), Où que (ce soit), Quelque N que (ce soit).

(3) Tu peux inviter qui que ce soit.

(4) Tu peux manger quoi que ce soit.

(5) Tu peux aller où que ce soit.

(6) Tu peux consulter quelque livre que ce soit.

Il y a deux contextes syntaxiques dans lesquels on peut trouver ces TCL concessifs :

1. En construction avec une proposition dans un complexe adjoint à valeur de proposition concessive comme dans (7). Tous les termes du paradigme connaissent ce fonctionnement.

(7) Quoi (que ce soit) qu'il fasse, on le critique.

2. En position d'argument d'un verbe principal (sujet, objet, complément circonstanciel) et sans être suivi nécessairement d'une proposition, comme dans (3-6). Seuls certains membres du paradigme (qui que ce soit, quoi que ce soit, où que ce soit) admettent ce fonctionnement.

Beaucoup de travaux ont proposé des typologies pour la distribution de cet item ( Gaatone 1971, Muller 2006, Tovena et al. 2004, Vlachou 2007). Nous nous intéresserons aux éléments qui, en fonction de GN, peuvent être couramment analysés comme termes de polarité négative et/ou comme termes de choix libre.

\subsection{Le comportement linguistique de quoi que ce soit.}

Ces expressions ne sont pas autorisées comme argument de phrases épisodiques positives, comme illustré en (8) :

(8) * Quoi que ce soit m'a déplu.

$2 \quad$ Il suffit, pour s'en persuader, de considérer une étude détaillée des restrictions de sélection sur any qui introduisent le travail de Horn (2005). Pour ne prendre que quelques exemples significatifs, on n'a pas d'emploi de quoi que ce soit comparable à "any two hours", à "He did not ask anyone, he asked his father", ou à "Pick any card".

Paul Reed (2000), explore en détail les différentes manières de rendre en français ce qui est exprimé par any en considérant un paradigme large ( quoi que ce soit, n'importe quoi, un $N$ quelconque, tout).

3 La forme Quel que soit n'a pas d'usage comme groupe nominal et nous la laissons pour cette raison un peu à l'écart. Elle relève cependant parfaitement de l'analyse "disjonctive" que nous proposerons pour les autres termes du paradigme. 
Cette propriété conduit à essayer de les ranger du côté des termes de polarité négative (TPN). Pour la plupart des locuteurs du français contemporain ${ }^{4}$, comme le signale Vlachou (2007), ils sont acceptables comme complément direct d'un verbe nié :

(9) Je n'ai pas mangé quoi que ce soit.

Ils sont également acceptables dans les autres contextes typiques qui sélectionnent les termes de polarité négative, protase de conditionnelle, questions et comparatives.

On reconnaît là un comportement qui l'apparente à l'anglais any. Et il y a beaucoup d'autres points de comparaison avec cette forme.

Le phénomène appelé "subtrigging" certains emplois en épisodique. (10) n'est pas très naturel, mais l'adjonction d'une relative rend la phrase plus acceptable en (11) :

(10) ? J'ai lu quoi que ce soit.

(11) J'ai lu quoi que ce soit qui pouvait être pertinent.

De même, les contextes de permission, typique des termes de choix libre, acceptent quoi que ce soit :

(12) Vous pouvez inviter qui que ce soit.

En revanche, la modalité devoir ne semble pas se combiner très heureusement avec quoi que ce soit, si on excepte les cas de subtrigging (cf. Muller 2006, Vlachou 2007, p. 210); (13) est peu naturel, (14) nettement meilleur :

(13) ? Vous devez manger quoi que ce soit.

(14) Vous devez manger quoi que ce soit que l'on vous propose.

Il y aussi des différences avec any. Le fonctionnement de quoi que ce soit avec l'impératif n'est pas admis :

(15) * Choisissez qui que ce soit.

\subsection{Les analyses de quoi que ce soit}

Ces formes du français ont fait l'objet d'analyses détaillées dans Muller (2006) et Vlachou (2007).

$4 \quad$ Mais il y a des divergences sur ce point, dont le cas suivant est un témoignage assez curieux. Robert Martin, dans un ouvrage pédagogique et dans un passage sans aucun rapport avec ce sujet (Martin 2002) propose, comme exemple de phrase agrammaticale : "*Il ne critique pas quoi que ce soit". Bien que dans l'ensemble, les sujets français soient en désaccord avec ce jugement, nous en avons rencontré plusieurs, qui disent ne pas trouver parfaitement naturelle la combinaison de pas et de quoi que ce soit. Il serait trop long de discuter ce point en détail ici, mais il semble qu'il pourrait être éclairé par des considérations diachroniques. Une recherche rapide sur Frantext montre que quoi que ce soit a été très longtemps utilisé avec la forme archaïque de la négation (ne sans pas). Voici deux des nombreux exemples :" mais tout leur souci était de ne partager quoi que ce soit avec ma *Wenigkeit." (Henri Frédéric Amiel, 1866); "Je ne m' intéresse à quoi que ce soit de ce qui intéresse les autres." (Chateaubriand, 1848). Il se pourrait que de ce fait, certains locuteurs cultivés éprouvent des difficultés à admettre pas dans ce contexte.

5 Le phénomène ainsi désigné dans LeGrand (1975) s'applique à l'observation suivante : certaines phrases, dans lesquelles any n'est pas acceptable, deviennent nettement meilleures si on adjoint une relative : ? I read any book/ I read any book recommended for the course. 
Muller (2006) considère deux emplois de la forme, comme terme de polarité négative (TPN concurrent de personne, rien) et comme terme de choix libre, à comparer à n'importe quoi. A propos des termes de choix libre (TCL), il affirme que "beaucoup de ces constructions prennent des interprétations universelles", la forme étant paraphrasable par "tout" ou par un équivalent, mais note que "cependant, certains emplois ne peuvent pas s'analyser avec une valeur universelle (op. cit. , p. 18).

\section{2.1 Quoi que ce soit est-il ambigu?}

Je voudrais montrer rapidement, en préalable à la discussion des seuls emplois TCL, qu'il est quasiment impossible d'assigner à l'item, comme signification, l'alternative (16):
(16) Quoi que ce soit
Existentiel (TPN)
$=$ une chose
Universel (TCL)
$=$ toute chose

C'est pourtant une tentation assez forte, au vu de contrastes comme (17)/(18):

(17) Je n'ai pas dit quoi que ce soit.

$=$ je n'ai pas dit une parole.

(18) Quoi que ce soit peut s'avérer un problème.

$=$ tout peut s'avérer un problème.

Mais une fois que l'item serait muni de ces deux significations, comment expliquer qu'il ne puisse être universel dans les contextes où il peut être TPN? ${ }^{6}$ Ainsi, la phrase (19) devrait être ambiguë :

(19) Je n'ai pas dit quoi que ce soit.

a. Je n'ai pas dit une (seule) chose.

b. Je n'ai pas dit tout.

Or l'interprétation b. n'existe pas.

On se trouve donc devant un dilemme: quoi que ce soit a parfois la valeur d'un quantificateur universel, mais en d'autres contextes, il semble ne pas pouvoir s'analyser comme tel.

Pour résumer les données, quoi que ce soit est régi par les contraintes suivantes :

1. il est légitime dans tous les contextes légitimant les TPN et dans ces contextes ne peut s'interpréter que comme TPN;

2. il n'est pas correct dans les épisodiques, sauf cas de subtrigging;

3. dans tout autre contexte que les TPN où il est acceptable, quoi que ce soit est associé à une interprétation universelle.

\subsubsection{L'élargissement.}

Vlachou (2007, p. 145) définit quoi que ce soit comme un TCL indéfini d"'élargissement" (du terme "widening" introduit par Kadmon \&Landman (1993) pour any). L'idée d'élargissement note le fait qu'une variable est contrainte de parcourir toutes les alternatives, même les moins typiques.

Le point important est que cette valeur d'élargissement est commune aux emplois comme TPN et aux emplois comme TCL; (20) semble intuitivement plus fort, et exclut davantage les exceptions éventuelles que (21):

(20) Qui que ce soit peut voir ce dossier.

(21) Tout le monde peut voir ce dossier.

$6 \quad$ C'est du moins vrai pour la forme quoi que ce soit sans relative. L'ajout d'une relative dans les contextes à interprétation TPN autorise l'ambiguïté : Je n'adhère pas à quoi que ce soit qu'il affirme peut avoir les deux interprétations. Nous reviendrons sur ce point très important dans la suite de l'article.

Un relecteur suggère que les phrases négatives permettent de confirmer ce point:

Ce dossier ne peut pas être vu par qui que ce soit. (= par personne). 
La notion d'élargissement, telle qu'elle est introduite par Kadmon \& Landman (1993) pour any, n'est qu'une partie de leur théorie. Ils ajoutent que any est légitime seulement si son usage donne matière à une information "plus forte" que ne produirait en sa place un indéfini dépourvu de cette valeur d'élargissement. Ils utilisent cette propriété, dite "renforcement", pour expliquer que any ne soit pas acceptable dans certains contextes. Les TPN, si on adopte une analyse assez bien admise depuis Ladusaw (1979) étant légitimés dans des contextes monotones décroissants, ces mêmes contextes garantissent que l'élargissement produira un énoncé plus fort qu'un indéfini simple dans ce même contexte (voir Vlachou 2007, p. 38-39).

Peut-on traiter quoi que ce soit avec la théorie de Kadmon \& Landman pour any?

Comme le dit très justement Vlachou, cette théorie est une théorie de la légitimation de any; quant à la sémantique de l'item, elle est simplement supposée être celle de l'indéfini, telle qu'elle est admise par la sémantique dynamique contemporaine : l'indéfini (Heim 1982) introduit une variable, et son contexte est responsable de la quantification de cette variable. Pour les emplois universels, il ne suffit plus de dire que quoi que ce soit introduit une variable, il faut expliquer pourquoi cette variable s'interprète dans la portée d'une quantification universelle. Pour Kadmon \& Landman, il en est ainsi dans les cas où l'interprétation de l'indéfini est générique. Cette possibilité serait ouverte pour l'indéfini any comme elle l'est pour l'indéfini $a$ :

"The sole difference between PS any and FC any lies in the interpretation of the indefinite NP : in the case of FC any, it is an indefinite interpreted generically." (op. cit, p. 374).

Cette proposition soulève plusieurs difficultés. Nous allons considérer ici seulement les difficultés liées à l'application à quoi que ce soit, sans discuter le fait qu'elles concernent ou non any. Le point principal de notre critique est que l'interprétation universelle de quoi que ce soit s'observe dans toute une série de contextes dans lesquels l'interprétation générique de l'indéfini un ne peut pas se produire.

1. Les contextes de légitimation de l'indéfini générique.

Le fait est bien connu, l'indéfini un ne peut recevoir une interprétation générique dans toutes les positions syntaxiques. Ainsi, il est quasiment impossible d'avoir un indéfini générique objet direct du verbe. Or il ne semble pas rare de trouver des phrases où quoi que ce soit en position d'objet est interprété universellement, comme en (22):

(22) Nous examinons quelque indice que ce soit, dans cette affaire. Nous contrôlons qui que soit, et nous verbaliserons qui que ce soit.

Les versions de ces phrases obtenues en substituant un $X$ à quoi que ce soit ne peuvent en aucun cas recevoir une interprétation universelle.

\section{Subtrigging.}

De même, autre fait connu, l'ajout d'une relative à quoi que ce soit améliore considérablement l'acceptabilité, et déclenche une interprétation universelle de la phrase. Or il n'en est rien pour l'indéfini, contraste illustré en $(23) /(24)$ :

Ce dossier ne pas être vu par tout le monde. (= certains peuvent, d'autre non).

Je partage parfaitement le jugement sur ces phrases négatives, mais l'origine de la différence me semble plutôt être une question de portée : qui que ce soit prend portée sur la négation, alors que tout est interprété dans la portée de la négation. Nous revenons infra sur ce point très important. 
(23) L'an dernier, j'ai lu quoi que ce soit qui a été publié en France sur les araignées.

(24) L'an dernier, j'ai lu un article qui a été publié en France sur les araignées.

C'est seulement en (23) que l'adjonction de la relative est responsable de l'acceptabilité, et c'est en (23) que cette adjonction est associée à une interprétation universelle. (24) persiste à n'offrir qu'une interprétation spécifique.

\section{Sur quelques restrictions combinatoires.}

Sans exception.

L'expression est remarquable car elle est, en quelque sorte, la glose de ce que Kadmon \& Landman postulent quant au sens lexical de any:

"What is it that any adds to the meaning of the indefinite NP? We think it contributes what we have described above as reduced tolerance to exceptions."

Kadmon et Landman (1993, p. 359).

Le point frappant est que cette expression se combine avec quoi que ce soit mais non avec un $N$ :

(25) Qui que ce soit, sans (aucune) exception, devra remettre ce devoir.

(26) Un étudiant (*sans exception) devra remettre ce devoir.

Intuitivement, sans exception semblerait sélectionner les items lexicaux qui s'interprètent comme quantification universelle ce qu'illustrent (27)-(30) :

(27) Tout le monde, sans exception, devra rendre le devoir.

(28) *La plupart des étudiants, sans exception, devront rendre le devoir.

(29) * Cinq étudiants, sans exception, devront rendre le devoir.

(30) Aucun étudiant, sans exception, ne rendra le devoir.

Au minimum, il s'agit d'une difficulté pour une analyse qui postulerait que les expressions qui que ce soit et un représentent la même catégorie sémantique (indéfini).

La combinabilité de quoi que ce soit et de sans exception en emploi de TPN n'est pas impossible, même si elle est un peu plus contrainte :

(31) Si vous parlez à qui que ce soit, sans exception, vous serez renvoyé.

(32) Il est plus malin que qui que ce soit, sans exception.

(33) ? Je n'ai pas mangé quoi que ce soit, sans exception

(34) ? Avez-vous quoi que ce soit, à me reprocher, sans exception.

\section{Presque.}

La combinaison de presque et de quoi que ce soit en usage de TPN n'est pas licite, le constat est bien admis dans la littérature (voir par exemple Deprez et Martineau 2006).

Pour ce qui est de l'usage "universel", on trouve sans difficulté des attestations :

(35) Presque quoi que ce soit peut être éclipsé par quelque chose de plus mauvais. - une question d'échelle.

\section{Google}

On en trouve cependant assez peu, et quasiment aucune pour qui que ce soit.

C'est une petite différence de distribution avec l'anglais any, qui semble admettre plus libéralement cette combinaison.

La conclusion qui se dégage de ces observations est que la combinabilité de quoi que ce soit avec sans exception et avec presque, qui l'oppose radicalement à l'indéfini générique régulier un $N$, est un argument contre l'assimilation de quoi que ce soit "universel" à une interprétation d'indéfini générique. ${ }^{8}$

$8 \quad$ Evangelia Vlachou (c.p.) objecte que la généricité n'est pas un trait sémantique d'item lexical, mais plutôt la propriété d'un usage, ou d'une construction. La combinabilité à d'autres 
L'intuition selon laquelle quoi que ce soit implique un élargissement du domaine de quantification à tous les individus, même les moins typiques, semble bien être vérifiée pour tous les emplois (choix libre et polarité). Mais pour ces emplois de choix libre, le problème principal est d'expliquer pourquoi ils sont des quantificateurs universels "forts". Si on parvenait à résoudre ce problème, on pourrait tenter d'intégrer les emplois de polarité comme cas particuliers d'usage d'une quantification universelle de portée maximale, en utilisant le schéma qui suit, illustré pour la négation,

(36) Je n'ai pas mangé quoi que ce soit

$$
\forall \mathrm{x} \neg \text { (j'ai mangé } \mathrm{x} \text { ) }
$$

quoi que ce soit étant ici analysé comme un quantificateur universel à portée large sur la négation. Il resterait à expliquer pourquoi cela n'est possible que pour les légitimeurs de TPN, mais cela ne semble pas insurmontable. Nous y reviendrons.

Vlachou (2007) retient la notion d'élargissement comme propriété cruciale de quoi que ce soit, et la thèse que l'expression est indéfinie (deux points communs avec l'analyse de Kadmon \& Landman pour any).

Elle définit quoi que ce soit comme un TCI d'élargissement sur la base de la définition (37), qui, comme le signale l'auteur, est plus forte que la notion originale :

(37) Les indéfinis d'élargissement . Vlachou (2007)

a. un indéfini TCL d'élargissement sélectionne une valeur dans un ensemble plus large qu'un indéfini régulier. Cet ensemble contient toutes les alternatives utilisées par l'indéfini régulier, et se trouve élargi pour inclure toutes les alternatives possibles, pertinentes ou non.

b. Toutes les alternatives de cet ensemble élargi, sans exception peuvent être la valeur du TCL.

L'élément (b) discuté par Vlachou (op. cit. p. 118) est une adjonction à la notion originale. Ma propre interprétation de cette contrainte est qu'elle semble bien capter une quantification universelle sur un domaine élargi, bien que la formulation ne soit pas aussi claire, et que l'auteur maintienne que quoi que ce soit est un indéfini (non un quantificateur). La proposition est cependant illustrée sur un exemple qui accepte une formulation en termes de quantification universelle . (38) est ma propre reformulation :

(38) I don't have any potatoes

= a. sur l'ensemble élargi des pommes de terre (pertinentes ou non),

b. Pour chacun de ses éléments $\mathrm{x}$, nous ne possédons pas $\mathrm{x}$

L'application de ces théories à quoi que ce soit met bien en avant l'élargissement de son domaine de quantification. Mais si quoi que ce soit est traité comme un indéfini, une simple variable, ses interprétations universelles doivent être expliquées autrement.

Nous restons donc avec une question : d'où vient la valeur universelle de quoi que ce soit? Est-elle inhérente (présente en tout emploi) ou occasionnelle? Nous allons essayer de répondre à ces questions.

items ne serait donc pas forcément pertinente. J'accorde volontiers le premier point, mais non le second. Il revient à toute théorie qui traite un et quoi que ce soit comme des indéfinis (de simples variables) accédant à l'universel via une quantification générique, d'expliquer pourquoi on observe ces différences de combinabilité avec des expressions comme sans exception. Rien n'est sans doute impossible mais un gros avantage d'une analyse de quoi que ce soit comme quantificateur universel et de un comme simple variable est de n'avoir ici rien à expliquer. 


\section{La composition de quoi que ce soit.}

Si l'on a décrit jusqu'à présent quoi que ce soit comme un item lexical, pour en étudier le comportement, il est indiscutable que l'expression est complexe, analysable, et il est de bonne méthode d'essayer d'expliquer le comportement d'un tout par la nature et la structure de ses éléments.

\subsection{Le tour suivi d'une relative.}

Les expressions étudiées apparaissent très librement avec une expansion relative :

Les constructions Qu-que ce soit + relative

Quoi que ce soit dont il parle

Qui que ce soit que j'aime

Lacunes dans la distribution en $\mathrm{FM}^{9}$

Où que ce soit que j'aille

*Quand que ce soit qu'il vienne

Pour qui que ce soit qu'il vote

L'analyse syntaxique de ces complexes peut être esquissée sans faire le choix d'une grammaire particulière, pourvu que l'on accepte la terminologie courante et une très légère variation de l'ordre des mots consistant à laisser quoi dans la position vers laquelle toutes les théories syntaxiques le mettraient en relation.

\section{Structure des constructions Qu-que ce soit +relative}

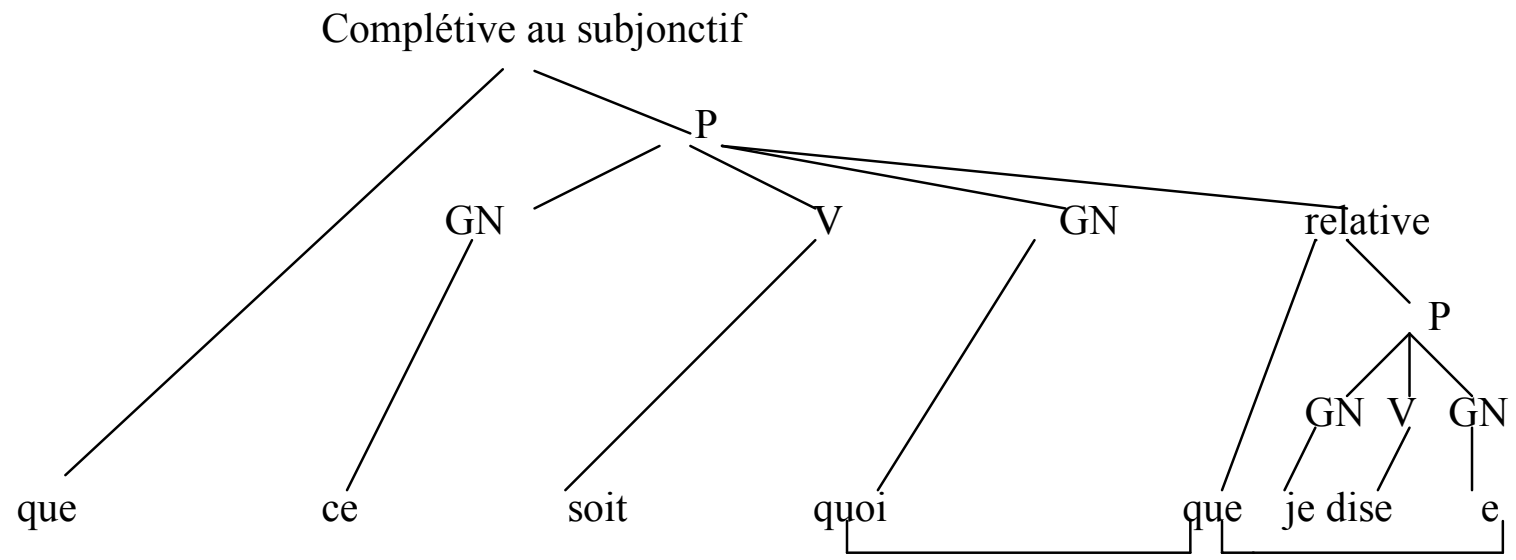

La seule particularité supposée est que le segment initial dans quoi que ce soit est un relatifinterrogatif attribut du sujet $c e$,"monté" en position initiale. Beaucoup d'analyses syntaxiques, je pense, admettraient la relation illustrée par la figure (41) :

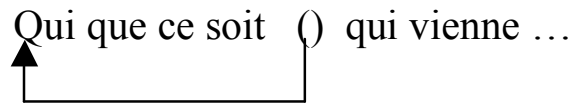
9 Sur l'histoire et la constitution de ce paradigme du français, voir la très intéressante
étude de M. Pierrard (2009). 
D'autre part, la structure "canonique", sans montée du relatif interrogatif en tête de phrase (*Que ce soit qui qui vienne), n'existe pas en français. Il est donc tentant de dire que cette structure n'existe que sous la forme avec montée de qui en position initiale: Qui que ce soit qui vienne.

De tels rudiments d'analyse (indépendamment de leur implémentation sérieuse dans une syntaxe particulière) donnent correctement les catégories et fonctions syntaxiques des éléments composants.

Ils suffisent aussi à indiquer que la "montée" en position initiale du relatif-interrogatif peut laisser planer un doute sur la catégorisation de ces structures : s'agit-il de phrases de structure Que P, comme les structures canoniques correspondantes (Que ce soit Pierre ou Marie qui vienne), ou de $G N$ interrogatifs (qui/quoi) modifiés par une structure phrastique, et acceptant donc les distributions de GN?

En réalité, ce que nous observons, c'est que de telles structures sont ambiguës syntaxiquement, et connaissent les deux distributions :

Que P Quoi que ce soit qu'il dise, Marie est admirative.

GN Marie admire quoi que ce soit qu'il dise.

Nous observons aussi que la construction admet souvent des variantes "courtes" comme en (42) et (43) :

(42) Quoi qu'il dise, Marie est admirative.

(43) Marie admire quoi qu'il dise. ${ }^{10}$

Le jugement des locuteurs est en général que la variante courte est plus naturelle en distribution que $P$ qu'en distribution de GN. Certains admettent cependant parfaitement la phrase (44), où la variante courte est sujet :

(44) Quoi qu'il fasse me ravit.

\subsection{La présence de "ce soit- $x$ qu".}

La grammaire du français autorise en principe à instancier un GN par un pronom relatif interrogatif, et les phrases en que $P$ ne dérogent pas à ce principe. En revanche cette construction connaît des restrictions sur la montée de l'interrogatif en position initiale de la structure ; où et quoi montent sans difficulté en français moderne :

(46) Quoi que Pierre regarde.

(47) Où que Pierre aille.

Les autres cas sont moins acceptables:

(48) ? Qui que Pierre regarde, Marie est furieuse.

(49) ?? Qui que regarde Marie, Pierre est furieux.

(50) * Qui qui embrasse Marie, Pierre est furieux.

Le tour avec ce soit autorise en revanche la montée dans tous les cas :

(51) Qui que ce soit que Pierre regarde, Marie est furieuse.

(52) Qui que ce soit que regarde Marie, Pierre est furieux.

(53) Qui que ce soit qui embrasse Marie, Pierre est furieux.

Pour résumer ces observations, la structure longue de type quoi que ce soit + relative est syntaxiquement une subordonnée en que $P$ avec montée initiale d'un interrogatif.

10 Cette phrase n'est pas admise par tous les locuteurs comme nous le signale un de nos relecteurs. 
La version courte (quoi que Pierre fasse, où que Pierre aille) n'est pas acceptable pour tous les interrogatifs (?Qui qui embrasse Marie) et paraît, à cette réserve près, fonctionner de la même manière que la forme longue. Il est également notable qu'elle est mieux acceptable en français moderne dans une distribution de type que $P$ que dans une distribution de type GN.

\subsection{Sémantique des constructions $Q u$-que ce soit qu+relative.}

La sémantique de ces constructions a pour élément crucial la sémantique du relatif interrogatif qui fait l'objet de la montée en position initiale, et elle existe sans relatif, mais uniquement dans les constructions utilisant une disjonction, illustrées par (54) et (55) :

(54) Que Pierre ou Marie vienne

(55) Que ce soit Pierre ou Marie qui vienne

Il s'agit de complétives standard au subjonctif dont la seule particularité est qu'un argument est instancié par une disjonction ( $a$ ou $b$ ). Ce seul facteur détermine des comportements qui n'existent pas pour une complétive sans disjonction comme (56) :

(56) Que ce soit Pierre qui vienne

La disjonction ouvre la possibilité d'une distribution propre qui n'existe pas pour les phrases dépourvues de disjonction, distribution illustrée par (57) :

(57) Que ce soit Pierre ou Marie qui vienne, fuyez.

*Que ce soit Pierre qui vienne, fuyez.

La syntaxe de ces subordonnées à disjonction est spéciale : une subordonnée de forme que $P$ est détaché et dépourvue de tout lien anaphorique avec la principale. Le lien syntaxique est donc différent de ce que l'on observe pour (58), mais aussi pour (59):

(58) Que ce soit Pierre qui vienne, Marie le souhaitait.

(59) Que ce soit qui vienne et Marie sautait de joie.

En effet, la proposition initiale de (57) relie la proposition en que $P$ à un impératif, ce qui est incompatible avec la présence d'un et.

Le point à mettre en avant est que si la subordonnée est disjonctive, sans exclure une anaphore (cf. 58), ni l'anaphore ni une relation en "et" ne sont nécessaires pour que la phrase soit correcte et interprétable.

La sémantique de ces complétives disjonctives est spéciale; elle peut être paraphrasée par : Que $P Q=$ Pour chacune des alternatives introduites dans $\mathrm{P}$, sans exception, $\mathrm{Q}$.

Le passage de la structure lexico-syntaxique à la sémantique est loin d'être transparent :

- Il y a bien une expression qui évoque des alternatives : "ou";

- il n'y aucun mot qui soit à la source de la quantification universelle (sans exception) contenue dans l'interprétation finale.

Il y donc là un problème d'interface syntaxe/sémantique, indépendant de l'analyse ou de l'existence de quoi que ce soit, et que l'on appellera le problème des complétives disjonctives.

\subsubsection{Le problème des complétives disjonctives}

Ce problème n'est pas indépendant de l'analyse de quoi que ce soit :

a. la plupart des analyses syntaxiques établiront une relation entre quoi que ce soit et les complétives au subjonctif.

b. La plupart des analyses sémantiques font un lien entre les relatifs interrogatifs et les disjonctions. 
c. On est obligé de constater que $Q U$ - que ce soit qu-déclenche le même type de construction spécifique que les complétives disjonctives, comme en (60) :

(60) Qui que ce soit qui vienne, partez.

Pour les complétive disjonctives particulières, par exemple, on observe comment le contexte peut préciser une relative absente. Considérons l'exemple (61):

(61) A- Qui viendra?

B- Que ce soit Pierre ou Marie, ça m'est égal.

$=$ Que ce soit Pierre ou Marie [qui vienne], ça m'est égal.

Bien qu'aucune relative ne figure effectivement dans la réponse de $\mathrm{B}$, il est impossible

d'interpréter le dialogue sans utiliser le contexte pour restituer le contenu d'une relative (dans l'exemple, par pure répétition du prédicat de la question de A).

Dans le cas des disjonctives généralisées on observe des phénomènes comparables, illustrés par (62) et (63) :

(62) A- Qui viendra?

B- Qui que ce soit, fuyez

$=$ Qui que ce soit [qui vienne], fuyez.

(63) A-C'est Pierre ou Marie, qui viendra.

B- Qui que ce soit [qui vienne], fuyez.

Le point intéressant de l'exemple (63) est que c'est le contenu de la relative qui est spécifié par le contexte, non l'ensemble des alternatives; la phrase s'interprète comme : qui que ce soit (Pierre, Marie, ou autre) qui vienne, fuyez, et non comme (qui que ce soit de Pierre et de Marie).

La saturation d'une relative par son contexte semble être cependant une option limitée aux emplois "absolus" de $Q U$-que ce soit, emplois en tête de phrase à valeur concessive. Il est beaucoup plus difficile de constater ce phénomène si $Q U$-que ce soit se trouve en position de groupe nominal comme en (64):

(64) A- Pierre, Jean, Marie, viendront.

B- Qui que ce soit verra les dégâts. (/Je n'en parlerai pas à qui que ce soit).

La réplique de $B$ semble impliquer une totale généralité : qui que ce soit $=$ tout le monde, et pas seulement l'un des éléments de l'ensemble \{ Pierre, Jean, Marie\}, et pas seulement ceux qui viendront. Autrement dit, en position de GN, QU-que ce soit (sans relative) ne spécifie pas celle-ci par reprise d'un élément du contexte. Nous reviendrons sur ce point infra.

\subsubsection{La sémantique des complétives disjonctives.}

Revenons sur l'énoncé (57):

(57) Que ce soit Pierre ou Marie qui vienne, fuyez.

L'interprétation est proche des phrases (65) ou (66):

(65) Si Pierre ou Marie vient, fuyez.

(66) Si Pierre vient, fuyez, et si Marie vient, fuyez.

Pour reprendre les termes de Lewis (1975), la proposition initiale explicite un certain nombre de cas possibles (on dit aujourd'hui, en utilisant un anglicisme, des "alternatives") déterminant un restricteur ou domaine de quantification, et la phrase dans son ensemble affirme que pour chacun des cas, sans exception, la proposition principale est valide. En substance, la juxtaposition d'une proposition exprimant des alternatives et de la proposition principale s'interprète comme une quantification universelle assertant que pour chacune des alternatives, 
la proposition principale est valide. Il s'agit d'une quantification universelle forte, non d'une quantification universelle faible de type générique.

La grammaire, indépendamment de l'existence des expressions qui nous occupe, devra imputer cette sémantique aux complétives disjonctives du français. On trouve dans Muller (2006, p. 18) une analyse des emplois dits concessifs comme analyse du "schème syntaxique que $P$ Subj $Q$ dans lequel la proposition au subjonctif couvre un domaine d'oppositions". Muller y utilise un rapprochement explicite entre les disjonctions et la forme quoi que ce soit qui est très proche de ce que nous supposons ici.

Hadermann (2007, p. 121) citant Leuschner (2006, p. 19) mentionne en linguistique générale et comparative la catégorie des "concessive conditionals ", conditionnels qui relient un ensemble de valeurs pour l'antécédent à une valeur pour un conséquent unique, et les "universal concessive conditionnals" reposant sur un mot WH- et un préfixe d'indifférence ou une particule.

Ce que nous analysons ici s'inscrit bien dans ce paradigme : la valeur conditionnelle est apportée par la complétive détachée au subjonctif, la constitution d'un ensemble d'alternatives provient de la disjonction interne à cette complétive ou d'un relatif interrogatif en QUreprésentant une disjonction "généralisée". L'interprétation conditionnelle résulte de la juxtaposition d'un ensemble d'alternatives et de la principale, traitée comme association d'un restricteur et d'un scope nucléaire. Que la quantification soit universelle se justifie aussi bien (ou aussi mal) que dans le traitement proposé par Lewis pour les phrases en si : Lewis affirme en effet que les structures conditionnelles sans quantificateur exprimé sont en fait interprétées comme si elles contenaient un quantificateur universel. (voir Corblin 2002 pour plus de détails.) Ce qui reste peut-être à justifier dans ce cadre, c'est la valeur de quantification universelle "forte" de cette tournure, qui est différence de ce que l'on observe pour les conditionnelles en si.

En effet, les conditionnelles en $s i$ sont plutôt du côté des généralisations génériques, c'est-àdire de celles qui admettent des exceptions.

(67) A-S'il pleut je reste à la maison.

B- Toujours?

A- Non, mais en général.

En revanche, un trait remarquable des complétives disjonctives (et de quoi que ce soit)est d'affirmer très fortement que pour les alternatives couvertes, la principale vaut, sans aucune exception. Peut-être doit-on l'imputer au fait que les alternatives sont explicitement marquées comme telles par une disjonction. Quoi qu'il en soit, ce trait est commun aux complétives disjonctives et à quoi que ce soit et il s'agit donc d'une propriété de la construction.

P. Jacobson (1995) s'interroge sur la force quantificationnelle des relatives libres et propose d'interpréter ces relatives comme des GN définis référant à toutes les alternatives. Il me semble possible de rapprocher $Q U$ - ce soit qu-P et relatives libres, mais je doute qu'il y ait en français identité des mécanismes en ce qui concerne l'universalité "forte". Considérons (68) : (68) J'aime ce que vous faites.

La phrase implique bien que j'aime tout ce que vous faites, mais il s'agit d'une universalité faible, du même ordre que la généralité générique et beaucoup moins forte que l'universalité très forte de la version :

(69) J'aime quoi que ce soit que vous fassiez.

D'autre part, il semble préférable d'avoir une explication qui couvre en même temps les complétives disjonctives et quoi que ce soit. Notre proposition est donc que l'origine de l'universalité forte est dans la complétive au subjonctif disjonctive. $Q U$ - ce soit $q u-P$ hérite de cette propriété simplement parce qu'il s'agit d'une complétive disjonctive (généralisée). 


\section{Les emplois de quoi que ce soit sans relative.}

Dans la logique que nous développons ici, quoi que ce soit est vu comme une structure incomplète, à relative non-spécifiée. La non-spécification a deux conséquences :

1) syntaxique : le segment est catégorisé comme un GN, non comme une proposition.

2) sémantique : plus aucun élément explicite ne vient restreindre le domaine des alternatives.

Le point qui nous paraît important à souligner est qu'on a affaire à un tour sans relative où celle-ci n'est pas spécifiée et enrichie par le contexte. Il me semble très difficile de trouver des exemples probants où le contexte viendrait restituer une relative lorsqu'il s'agit d'un emploi en position de GN. Le contraste est en cela assez fort avec ce qu'on a observé pour les usages de l'expression en position d'adjoint détaché.

Essayons par exemple d'utiliser le contexte pour circonscrire l'interprétation à un domaine d'individus :

(70) A- As-tu interrogé les élèves de la classe?

B- Je n'ai pas interrogé qui que ce soit.

La réponse de $\mathrm{B}$ ne s'interprète pas, selon moi, comme : Je n'ai interrogé aucun élève de la classe. Elle s'interprète comme : je n'ai interrogé strictement personne, élève de la classe ou non. Le contraste est également saisissant avec un quantificateur ordinaire :

(71) A- As-tu interrogé les élèves de la classe?

B- Personne n'a rien vu.

La réponse de $\mathrm{B}$ ici s'interprète au contraire très naturellement comme: "personne au sein de la classe". Il est quasiment impossible également d'utiliser le contexte pour "récupérer" l'équivalent d'une relative.

(72) A-Considères-tu comme suspects ceux qui étaient présents?

B- Qui que ce soit peut avoir fait le coup.

Il est typique que la réponse de B quantifie sur un domaine plus large (tout le monde), et serait plutôt interprétée comme une réponse négative à la question de A. Là encore, le contraste est saisissant avec ce qu'on observe pour un quantificateur ordinaire :

(73) A-Considères-tu comme suspects ceux qui étaient présents?

B- La plupart peuvent avoir fait le coup.

On interprétera ici naturellement comme "la plupart de ceux qui étaient présents".

L'absence de la relative fait que le champ des alternatives considérées sur le domaine des individus n'est simplement limité par rien : quoi que ce soit (sans relative) implique comme alternatives l'ensemble maximal des individus. Nous continuerons d'appeler cette propriété "élargissement" et de postuler qu'elle est caractéristique des emplois de quoi que ce soit. Le tour sans relative quantifie sur tous les individus "possibles" appartenant à la catégorie (humain/chose). On retrouve ici la valeur "modale" mise en avant par Dayal $(95,98)$ dans son analyse de any.

\subsection{Les emplois universels de quoi que ce soit.}

L'emploi de quoi que ce soit en structure complète s'interprète comme une quantification universelle forte (sans exception) sur les alternatives caractérisées par la relative, laquelle constitue le restricteur du quantificateur universel associé.

La structure incomplète s'interprètera de même, comme quantification universelle forte sur le domaine maximal des individus. L'acceptabilité de l'énoncé est déterminée par la capacité du contexte à accepter une quantification universelle forte sur le domaine maximal.

La phrase (74) n'est pas acceptable : 
(74) ? Quoi que ce soit m'est tombé sur la tête.

Une épisodique génère en principe une clôture existentielle, et elle est d'autre part limitée pour des raisons de plausibilité à des domaines restreints. Elle est peu compatible avec une quantification universelle maximale: il est peu probable que toutes les choses du monde, sans exception, me tombent sur la tête.

En épisodique, une relative est indispensable pour rétablir l'acceptabilité comme en témoigne le contraste $(75) /(76)$ :

(75) *J'ai lu quoi que ce soit.

(76) J'ai lu quoi que ce soit qui concernait l'affaire.

L'ajout de la relative rétablit pour l'argument de lire une délimitation matérielle qui en fait un argument plausible d'une épisodique.

\subsection{Les emplois de quoi que ce soit classés comme TPN}

Les propriétés communes des emplois analysés comme TPN sont les suivantes :

a. Quoi que ce soit figure dans la portée syntaxique d'un opérateur logique.

b. Cet opérateur logique est monotone décroissant et légitime les termes de polarité.

c. Quoi que ce soit ne peut pas s'interpréter comme universel dans la portée de l'opérateur considéré.

Le fonctionnement standard de $Q U$-que ce soit qu- est "concessif conditionnel" : la relative fournit la disjonction des alternatives sur lesquelles opère un quantificateur universel fort. S'il est dépourvu de sa relative, on aura le même fonctionnement, mais sur le domaine maximal, puisque la spécification apportée par la relative n'est pas présente. Dans le fonctionnement de la construction complète, la relative est le restricteur de la conditionnelle et la phrase matrice est le scope. Cette analyse vient d'être appliquée aux emplois classés comme universels dans la section précédente. Elle peut en réalité être étendue aux emplois analysés comme TPN :

- Je n'ai pas mangé $\mathrm{x}=$ Pour tout $\mathrm{x}$, qu'il s'agisse de $\mathrm{a}$, de $\mathrm{b}$, de c, etc., sans exception, [je n'ai pas mangé $x$ ]

Cette analyse n'est en rien ad hoc dans notre approche. Elle est exactement celle que nous proposons pour les emplois de GN de la structure complète. Ainsi, pour l'exemple (77),

(77) J'ai noté quoi que ce soit qu'il ait dit.

l'hypothèse est que la phrase s'interprète exactement comme la structure conditionnelle comportant un quantificateur universel fort sur les alternatives (78) :

(78) Quoi que ce soit qu'il ait dit, je l'ai noté.

Nous ne faisons donc rien d'autre que d'accorder à quoi que ce soit la possibilité de s'interpréter comme quantificateur universel de portée maximale liant une variable dans sa position syntaxique d'origine.

Dans cette analyse, il faut expliquer pourquoi le fonctionnement de quoi que ce soit fait une différence cruciale entre variable dans la portée d'un opérateur décroissant (phrase correcte) et variable dans la portée d'un autre opérateur (phrase agrammaticale). Cette différence est illustrée par le contraste (79)/(80)-(81) :

(79) Je mange rarement quoi que ce soit le soir.

(80) * Je mange parfois quoi que ce soit le soir.

(81) * Deux fois par semaine, je mange quoi que ce soit le soir.

(80) comporte un opérateur croissant (parfois) et (81) un opérateur non-monotone (deux fois par semaine). 
Lorsque la phrase est acceptable, c'est seulement avec une interprétation où l'universel prend portée sur l'opérateur décroissant. La portée maximale est une propriété qui pour nous est générale pour tous les emplois de quoi que ce soit (sans relative). Le point à expliquer est que seul les opérateurs décroissants produisent une phrase acceptable dans ce cas.

Considérons ce qu'on observe pour les mêmes contextes dans les cas où une relative est présente. L'acceptabilité est rétablie en tout contexte :

(82) Je mange rarement quoi que ce soit que tu cuisines le soir. $(\Downarrow)^{11}$

(83) Je mange parfois quoi que ce soit que tu cuisines le soir. $(\Uparrow)$

(84) Peu de Chrétiens pratiquent quoi que ce soit que demande l'Evangile. ( $\Downarrow)$

(85) Beaucoup de Chrétiens pratiquent quoi que ce soit que demande l'Evangile. ( $)$

Cependant, les interprétations ouvertes ne sont pas tout à fait les mêmes. Pour les opérateurs décroissants, les deux hiérarchies de portée sont admissibles, et (84) a deux interprétations : 1) Universel >Opérateur : " Pour chaque chose, sans exception, demandée par l'Evangile, peu de Chrétiens la pratiquent."; 2) Opérateur > Universel : "Peu de Chrétiens pratiquent tout ce que demande l'Evangile". Pour un opérateur croissant comme beaucoup, la hiérarchie de portée relative qui s'impose fortement est Opérateur > Universel. Ainsi, (85) a pour interprétation préférée sinon exclusive: "Beaucoup de Chrétiens pratiquent tout ce que recommande l'Evangile".

Il ne semble pas y avoir de différence significative, en général, entre opérateurs décroissants et autres quant à la portée inverse d'un universel lexical: ${ }^{12}$

(86) Peu de gens aiment tous les tableaux de l'exposition.

(87) Beaucoup de gens aiment tous les tableaux de l'exposition.

La portée inverse (pour chacun des tableaux il y a peu/beaucoup d'admirateurs) est également difficile à obtenir pour le distributif chacun:

(88) Peu de gens aiment chacun des tableaux.

(89) Beaucoup de gens aiment chacun des tableaux.

Elle est peut-être un peu plus accessible dans ce cas, mais sans différence significative entre beaucoup et peu.

Si l'universel, en général, n'exclut pas strictement d'avoir portée inverse sur un opérateur croissant, nous n'avons que deux principes d'explication pour justifier que quoi que ce soit ne soit grammatical qu'avec un opérateur décroissant. Ou bien ce qui est en cause, c'est la quantification universelle forte attaché à quoi que ce soit dans tous ses emplois, c'est-à-dire le parcours du domaine sans exclure le moindre individu; ou bien ce qui est en cause, c'est la maximalité du domaine, laquelle est le propre de quoi que ce soit en emploi absolu, c'est-àdire le fait que le domaine doit inclure tous les individus possibles, sans aucune restriction.

Ce qui est crucial pour trancher, c'est d'évaluer de plus près ce qu'il en est des structures complètes, déjà illustrées par (84) et (85). Si aucune structure complète ne donne lieu à une phrase grammaticale avec un universel de portée large en dehors des opérateurs croissants, c'est la quantification universelle forte qui est le facteur déterminant, et le type d'explication proposé par Kadmon et Landman peut être emprunté tel quel. Si en revanche de telles

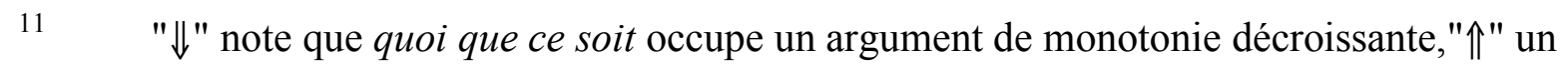
argument de monotonie croissante.

${ }_{12}$ Voir l'étude de J.-C. Anscombre (2006) sur les différences d'usage de tout, chaque, et n'importe quel.
} 
structures acceptent l'interprétation à portée large, le facteur déterminant est plutôt la maximalité du domaine.

La discussion est compliquée parce que, de manière générale (voir supra), la portée inverse d'un universel sur un opérateur croissant n'est pas une option préférée. Il semble cependant aisé de trouver des phrases qui illustrent ce cas, comme (90) ou (91) :

(90) Dix membres du club exactement sont inscrits à quoi que ce soit qui ait été proposé.

(91) Deux policiers ont interrogé qui que ce soit qui confirmait ses dires.

Pour moi, (90) recouvre deux situations possibles : a) 10 membres se sont inscrits à tout; b) chaque activité sans exception a vu l'inscription de dix membres. L'interprétation (b) est évidemment une interprétation de quoi que ce soit + relative à portée large. Pour (91) il n'est pas nécessairement impliqué que les deux mêmes policiers aient interrogé tous les témoins.

Ces données semblent être des arguments directs contre une corrélation entre monotonie décroissante et élargissement. (90) et (91) impliquent bien élargissement, si la propriété est lexicalement attachée à quoi que ce soit, ils sont basés sur des opérateurs croissants, et ils admettent une ambiguiité portée large/portée étroite de l'universel.

Elles incitent même à revenir sur les emplois absolus de quoi que ce soit. Le type d'explication proposé par Kadmon et Landman pour any repose sur des implications strictes, et ne devrait souffrir aucune exception fondée sur le contexte. Un opérateur est décroissant ou non, et sinon, le "renforcement" postulé ne pourra pas se produire, et cette impossibilité implique non-acceptabilité. On ne s'attend pas, dans cette optique, à trouver que l'acceptabilité dépend largement du contexte lexical, or c'est ce que l'on observe. Considérons le contraste $(92) /(93)$ :

(92) *Beaucoup de gens mangent quoi que ce soit.

*Pierre mange souvent quoi que ce soit.

(93) Beaucoup de gens critiquent quoi que ce soit.

Pierre critique souvent quoi que ce soit.

Les énoncés (92) sont fortement rejetés par les locuteurs, alors que les énoncés (93) sont acceptés sans difficulté. ${ }^{13}$ Or ils ne s'opposent que par la nature des actions considérées, ils sont en tous poins de leur structure sémantique identiques et quoi que ce soit est dans un contexte croissant. Ces données sont inattendues dans le cadre d'une corrélation stricte (propriétés algébriques/grammaticalité) du type de celles que proposent Kadmon et Landman pour any.

Une déduction naturelle est que la dimension pertinente est la maximalité des emplois absolus, non la quantification universelle sans exception. Quoi que ce soit implique une quantification universelle de portée large sur tous les individus possibles. Nous avons déjà expliqué la mal-formation d'un exemple comme (74) en postulant qu'un énoncé épisodique ne pouvait admettre d'argument quantifiant sur tous les individus possibles. Et il s'agit d'une propriété spécifique de l'universel quoi que ce soit d'être, en quelque sorte "immunisé" contre toute restriction contextuelle de son domaine de quantification, qui doit rester, par nature maximal. Un autre universel comme tout, par exemple, sera en revanche acceptable dans le correspondant de (74); "Tout m'est tombé sur la tête" est correct, dans la mesure où il quantifie universellement sur un ensemble contextuellement restreint.

13 Un relecteur met en cause que les locuteurs acceptent (93) sans difficulté. Peut-être concéderait-il au moins une différence d'acceptabilité entre (93) et (92), lequel est clairement mal formé. 
Nous proposons de considérer que cette seule contrainte est à la source de toutes les agrammaticalités observées. Si les contextes décroissants sont toujours des sites d'accueil favorables pour quoi que ce soit, c'est parce qu'ils produisent des énoncés compatibles avec une quantification universelle forte sur tous les individus possibles. Considérons un énoncé négatif comme "Je n'ai pas mangé quoi que ce soit". Il n'y a pas de difficulté à considérer qu'il s'interprète comme : pour tout x possible, je n'ai pas mangé $x$. Le positif correspondant implique une quantification existentielle de la variable, et n'est pas compatible avec une quantification universelle sur tous les objet possibles. Protase de conditionnelle, questions, compléments de comparative ont la même propriété : ils sont compatibles avec une quantification universelle maximale de la variable argument. ${ }^{14}$

Cette explication présente peut-être l'avantage d'introduire assez de flexibilité pour rendre compte de contrastes comme (92)/(93). Il est impossible, matériellement que des gens mangent toutes les choses possibles (même si on restreint aux choses comestibles); en revanche, on imagine mieux que des gens critiquent toutes les choses possibles.

\subsection{La portée large de quoi que ce soit.}

Dans la portée d'un opérateur décroissant, quoi que ce soit ne peut pas s'interpréter comme universel à portée étroite; (94) ne peut pas signifier je ne mange pas tout.

(94) Je ne mange pas quoi que ce soit.

Il est bien nécessaire de faire l'hypothèse que c'est l'absence de relative qui est déterminante, car sa seule adjonction dans le même contexte déclenche une ambiguïté :

(95) Je ne mange pas quoi que ce soit qu'il cuisine.

$=\mathrm{Je}$ ne mange pas tout ce qu'il cuisine.

$=$ Je ne mange rien de ce qu'il cuisine.

Quoi que ce soit semble donc perdre, s'il perd sa relative, la capacité de s'interpréter in situ comme un universel dans la portée d'un opérateur, et ne garde, comme option, que la possibilité de s'interpréter comme universel à portée large. Notons que les qualités de cet opérateur ne sont pas en cause, et que cela vaut également pour les opérateurs croissants; (96) ne peut pas signifier je mange parfois tout :

(96) *Je mange parfois quoi que ce soit.

C'est d'ailleurs la raison ultime selon moi de l'agrammaticalité de cette phrase : la seule option ouverte (portée large) est exclue pour les raisons évoquées ci-dessus, alors que dans la même position, la structure complète admet une interprétation à portée étroite, comme en témoigne (97), qui devient, de ce fait, acceptable :

(97) Je mange parfois quoi que ce soit qu'il cuisine, et cela lui fait plaisir.

Cette carte forcée pour l'interprétation universelle à portée maximale en l'absence de relative peut-elle être expliquée?

On peut certes en faire une propriété lexicale de l'expression quoi que ce soit traitée comme un segment figé. Mais si on compare l'item à la construction figée, cette propriété perdue n'est pas n'importe quelle propriété : l'absence de relative signifie que les alternatives associées ne

\footnotetext{
14 Beaucoup d'approches de any ont proposé des formulations qui semblent compatibles avec cette présentation, notamment Zwarts (1995) et Giannakidou (2001) en termes de non-véridicalité. C'est de l'approche de Dayal (1998) que l'analyse proposée ici semble la plus proche dans sa formulation. Jayez et Tovena 2010 (ce volume) défendent aussi des vues qui semblent aller dans le même sens, en admettant en particulier que "Les contextes monotones-décroissants permettent de s'abstraire des événements précis".
} 
reçoivent aucun contenu pour les restreindre et doivent être considérées comme maximales. Quoi que ce soit quantifie sur l'ensemble maximal des objets, pris en compte sans exception. Il y a donc une corrélation intéressante, qui se formule : si les alternatives couvertes sont maximales (sans relative), l'opérateur de quantification universelle ne peut avoir que portée large. Mais nous n'avons pas d'explication pour cette corrélation.

\section{Comparaison avec les traitements de any.}

Il est intéressant de confronter cette analyse de quoi que ce soit à la littérature sur any considérée un peu plus largement que ce que nous avons fait jusqu'à présent. Notre seul objectif ici est de voir en quoi ce qui est proposé pour quoi que ce soit rejoint, ou entre en opposition avec ce qui a été supposé pour any.

\section{1 Universel à portée large.}

Quoi que ce soit est bien analysé ici comme un universel à portée maximale, une analyse appliquée à l'anglais any par de nombreux logiciens et linguistes tels que Reichenbach (1947), Quine (1960), Horn (1972, chap. 3), Lasnik ((1972), Kroch (1975) et Eisner (1995). Nous reprenons en outre l'hypothèse que quoi que ce soit implique un élargissement du domaine de quantification de ce quantificateur universel, qui doit parcourir toutes les alternatives possibles. Cette hypothèse a été proposée par Vlachou (2007).

L'hypothèse de l'élargissement et du parcours de toutes les alternatives possibles a été faite pour l'anglais any par Kadmon \& Landman (1993) et par Dayal (1998) sous des formes légèrement différentes : les premiers parlent d'"élargissement suivant une dimension contextuelle", et la seconde de parcours de tous les "individus possibles ". Aucun de ces auteurs cependant ne combine pour any les traits universel, portée maximale et élargissement.

L'analyse de l'anglais any en emploi de choix libre qui se rapproche le plus de ce que nous proposons pour quoi que ce soit dans la période récente est celle de Dayal $(1998,2004)$. Elle présente des arguments établissant que l'explication de la valeur universelle de any ne peut pas être due à un opérateur générique (nous donnons des arguments du même ordre pour quoi que ce soit), et soutient que any est un universel qui s'oppose à every en ce qu'il quantifie sur le plus large domaine possible. L'auteur admet cependant que les emplois en contexte de polarité sont des emplois existentiels. Notre analyse postule en revanche, que quoi que ce soit est un universel de portée maximale en tout contexte.

C'est donc de l'analyse proposée pour any par Hintikka (1980) que nous sommes le plus proche :

(98) "any is unambiguous and it basically always has the force of a universal quantifier." Hintikka (1980, p. 101).

On trouvera dans cet article de nombreuses études d'exemples reposant sur la thèse que any est un opérateur de portée maximale, qui a priorité sur tout autre opérateur. Si nous ne nous prononçons pas sur la validité de cette thèse pour any, nous pensons qu'elle vaut pour quoi que ce soit.

\subsection{Arguments contre la thèse d'une analyse uniforme comme quantificateur universel.}

Vlachou (2007, p. 25) dont notre analyse s'inspire très largement, catégorise cependant quoi que ce soit comme un indéfini, et présente plusieurs arguments contre l'application d'une analyse comme quantificateur universel à any. Nous les considérons ici en tant qu'ils seraient 
opposables à notre analyse de quoi que ce soit.

1. Une approche de quoi que ce soit comme universel ne s'appliquerait pas à tous les contextes.

Dans ce qui précède, nous avons montré que les emplois dits de polarité s'analysent sans difficulté comme quantification universelle de portée maximale, comme illustré par (99):

(99) Je n'ai pas mangé quoi que ce soit.

$\forall \mathrm{x} \neg$ (j'ai mangé $\mathrm{x}$ )

Si une relative est présente, nous observons les deux possibilités d'interprétation universelle (étroite et large).

2. Quoi que ce soit n'aurait pas les mêmes relations de portée relative que les quantificateurs universels standard (en français tout, chaque, tous les).

Un argument de Giannakidou (2001) rappelé par Vlachou est que any (à la différence de every) ne peut prendre portée sur certains indéfinis.

(100) Some student will pick up any invited speaker from the airport.

La donnée empirique invoquée est que any dans cet exemple ne peut pas prendre portée sur some (la phrase implique donc un étudiant particulier). L'argument ne peut être étendu à quoi que ce soit, comme le montre (101) :

(101) Un policier devra contrôler qui que ce soit à l'entrée. ${ }^{15}$

Il ne me semble pas du tout imposé que (101) vise un seul policier et les deux hiérarchies de portée sont pour moi ouvertes.

3. Quoi que ce soit et la substituabilité en contexte de choix libre.

"[...] by analyzing any as universal quantifier, we cannot explain why any cannot be replaced by all in the following context":

Take any dress

\# Take all dresses"

Vlachou (2007, p. 26).

La donnée n'est pas facile à transposer en français car quoi que ce soit n'est pas très acceptable dans les impératives (sans relative) ${ }^{16}$. Ce serait donc la première chose à expliquer.

Mon explication de la mauvaise acceptabilité est que quoi que ce soit est trop peu spécifié et de trop large extension pour faire l'objet d'une demande d'action. Dès qu'une spécification intervient, la phrase redevient acceptable, comme en (102) :

(102) Choisissez qui que ce soit de compétent.

L'interprétation de quoi que ce soit est identique à celle de tous si le verbe n'implique rien quant au nombre d'individus à retenir.

(103) A- Qui devons-nous garder dans l'entreprise?

B- Gardez dans l'entreprise qui que ce soit qui acceptera le nouveau règlement.

$=$ Gardez dans l'entreprise tous ceux qui accepteront le nouveau règlement.

Ce qui semble particulier, dans l'exemple classique anglais (pick any card, take any dress), c'est le fait qu'il s'agit de prendre une (seule) robe/carte, ce qui est difficilement compatible, à

15 Evangelia Vlachou (c.p.) m'indique que plusieurs locuteurs ne partagent pas ce jugement. Ces locuteurs devraient refuser la phrase suivante : "Une signature doit être apposée par qui que ce soit sur son passeport". Elle est acceptable pour moi.

Ce point est relevé dans Muller (2007, p.89), qui note que les seuls emplois naturels ont des interprétations de "choix répété", ou impliquent une conditionnelle cachée. Je partage les jugements et les analyses de Claude Muller sur ce point. 
première vue, avec une interprétation de any comme quantificateur universel.

Mais il y a au moins un fait de l'anglais qui devrait être pris en compte dans la discussion : Il y a des phrases où any à l'impératif est clairement universel :

(104) Confiscate any liquor.

Dayal (1998)

On pourrait penser que l'interprétation est, dans la majorité des cas, universelle. Si la sémantique du verbe (pick, choose) présuppose une sélection d'individu unique dans un ensemble, elle est cependant incompatible avec l'interprétation universelle, sauf s'il est demandé de choisir un élément parmi les autres en répétant l'opération indéfiniment ${ }^{17}$, comme dans (105) :

(105) Pick any card. You will find that its back is similar to the others.

Dans un tel cas, l'interprétation est proche d'une conditionnelle : "If you pick any card, You will find...", et elle est compatible avec une interprétation universelle de any. Cependant, il est difficile de l'interpréter comme un ordre d'avoir à prendre chacune des cartes.

Mon intuition est que les exemples classiques réputés exclure l'interprétation universelle sont en fait universels, même s'ils ne demandent pas de choisir effectivement chacune des cartes pour que l'ordre soit satisfait. On pourrait les voir comme des restricteurs de conditionnelles de format :"Pour chacune des cartes sans exception, si vous la prenez, vous obéissez à mon ordre".

Pour ce qui est de quoi que ce soit, s'il est employé en impérative, il est indéniable qu'il a des emplois universels, comme en (106) :

(106) Fouillez qui que ce soit.

$=$ fouillez tout le monde.

Un verbe comme choisir, cependant, reproduit les mêmes effets d'unicité que l'anglais any. (107) Choisissez qui que ce soit, peu importe.

La même explication pourrait être invoquée : "choisir" impose une sélection sur un domaine d'individus, et on ne peut exécuter correctement l'ordre en sélectionnant chacun des individus du domaine. Avec un tel verbe, l'acceptabilité sera meilleure si la phrase peut être interprétée comme une conditionnelle d'interprétation : pour chacun des $x$, si vous choisissez $x$, peu importe. $^{18}$

Un argument du même ordre, rappelé par Horn (2005, p.186) est dû à Fauconnier (1979, pp. 297-298) : il semble à première vue difficile d'accepter que "I wonder if Susan married anybody" s'interprète effectivement comme $\forall x$ (I wonder if Susan married $x$ ); la phrase s'interprète plutôt comme $I$ wonder if $\exists x$ (Susan marry $x$ ).

L'argument est transposable au français qui que ce soit, car la phrase "Je me demande si Marie a épousé qui que ce soit" est acceptable pour de nombreux locuteurs. Il ne s'agit certes plus d'un impératif, mais encore une fois d'un verbe qui présuppose l'unicité de l'argument (to marry someone).

Comparons avec ce qu'on observe pour un verbe sans un tel présupposé, par exemple dans : "Je me demande si la police a contrôlé qui que ce soit". Apparaît immédiatement une interprétation universelle à portée étroite : Je me demande si $\forall x$ (la police a contrôlé x). En principe cette interprétation est un petit problème, non pour la thèse de l'interprétation universelle, bien sûr, mais pour la thèse de la portée large obligatoire (l'universel est interprété

17 Voir la notion de "choix répété" utilisée par Muller (2007). Cf. note précédente. 18 Une étude très détaillée du contenu conceptuel de la notion de "choix libre" est proposée dans l'article de J. Jayez et L. Tovena (ce volume). Cette analyse n'est pas cruciale pour l'étude de quoi que ce soit, puisque cet item, précisément, n'est pas excellent dans les emplois les plus typiques, lesquels utilisent plutôt "n'importe qui/quoi/quel" en français. 
ici dans la portée de $s i$ ). Il semble s'agir d'un point mineur qui distingue qui que ce soit (humain) de quoi que ce soit (non-humain), cf. infra.

Mais il y a aussi une autre interprétation (= je me demande si la police a contrôlé une seule personne) qui pourrait valider la transposition de l'argument de Fauconnier contre une analyse universelle. En réalité, je ne pense pas que cela soit exact. La représentation postulée par notre analyse est $\forall x$ Je me demande si (la police a contrôlé $x$ ); elle me semble fournir exactement le contenu de l'interprétation recherché : s'il est vrai que de chaque individu, sans exception, je me demande si la police l'a contrôlé, cela implique logiquement que je me demande si la police a interrogé une seule personne. Décider que l'inverse est vrai est problématique dans la mesure où se demander est un contexte opaque. Mais il paraît légitime au moins de traiter l'interprétation existentielle à la Fauconnier-Horn (Je me demande si $\exists x$ (la police a contrôlé $x$ ), et selon sa propre conception des contextes opaques comme une équivalence, une implicature ou une inférence de la représentation universelle postulée.

Il ne semble donc pas que ces cas fournissent d'argument décisif contre la thèse que quoi que ce soit s'interprète en tout emploi comme un universel de portée maximale.

\subsection{Les restrictions de sélection et la thèse de la quantification universelle pour tous les emplois.}

Il est vrai que presque fait une différence entre les emplois de TPN et les autres : il est inacceptable pour les premiers et acceptable pour les seconds :

(108) Presque qui que ce soit a accès à internet.

(109) *Je n'ai pas parlé à presque qui que ce soit.

(110) *Si vous avez besoin de presque quoi que ce soit, appelez-moi.

Il faut sans nul doute expliquer ce point, mais presque, le fait est reconnu dans la littérature, n'est pas un test très décisif : beaucoup de termes qui ne sont pas universels l'acceptent, comme les numéraux, par exemple. Ne pas se combiner avec «presque » peut donc difficilement passer pour une preuve absolue que l'on n'est pas universel.

Le test de "sans exception", qui semble mieux établir la nature universelle d'une expression, indiquerait que tous les emplois sont universels :

(111) Qui que ce soit, sans exception, peut consulter son fichier.

(112) Si vous laissez entrer qui que ce soit, sans exception, vous aurez des problèmes.

(113) Pierre s'est abstenu d'inviter qui que ce soit, sans exception.

Dans l'ensemble, il ne semble pas y avoir d'argument décisif pour réfuter la thèse que tous les emplois de quoi que ce soit sont universels.

\subsection{Arguments contre la portée maximale de la quantification universelle.}

La thèse de la portée maximale de la quantification universelle associée à qui que ce soit doit être confrontée à des exemples inspirés de Giannakidou (2001), dans lesquels un indéfini spécifique peut prendre portée sur quoi que ce soit :

(114) Un (de mes) étudiant(s) accompagnera qui que ce soit à l'aéroport.

L'indéfini initial peut dans ce cas avoir portée sur qui que ce soit, bien que cela ne soit pas obligatoire (voir supra la discussion des exemples (100) et (101)). Mais l'indéfini est un quantificateur particulier; pour certaines théories ce n'est pas (toujours) un quantificateur, et il a des emplois référentiels.

Notre approche ne prête, d'autre part, portée maximale qu'à la forme quoi que ce soit (privée de relative et de toute restriction contextuelle de cette relative). Pour certains exemples, il peut y avoir un doute, précisément si on a à l'esprit une restriction à un domaine particulier. 
Considérons (115) :

(115) Il ne répond pas à qui que ce soit

Hors de tout contexte, l'universel a portée large (= Il ne répond à personne). Supposons que le contexte impose un domaine restreint comme en (116) :

(116) A- Et ces lettres de demande d'emploi? Il y répond en général?

B- Il ne répond pas à qui que ce soit, mais la plupart des candidatures sérieuses ont une réponse.

Dans un tel cas, si on interprète qui que ce soit dans la portée de la négation, c'est que le contexte a fourni une restriction qui joue le même rôle qu'une relative et autorise

l'interprétation à portée étroite.

De manière générale, il faut constater que qui que ce soit admet, bien plus facilement que quoi que ce soit, de ne pas prendre portée large. Ce contraste est illustré par $(117) /(118)$ :

(117) Ils ne contrôlent pas qui que ce soit après huit heures.

(118) Ils ne mangent pas quoi que ce soit après huit heures.

La phrase (117) s'interprète très facilement comme "ils ne contrôlent pas tout le monde", et comme "Ils ne contrôlent personne; la phrase (118) ne s'interprète guère que comme : "Ils ne mangent rien"; l'interprétation "Ils ne mangent pas (de?) tout" semble forcée à la plupart des locuteurs.

Comment expliquer cette bizarre dissymétrie? Loin de constituer une objection pour notre approche, elle semble en découler assez naturellement. Rappelons que si une relative est présente, la portée étroite est toujours autorisée ; la portée large ne devient quasi-obligatoire que si cette relative est absente. Nous avons noté cette corrélation entre extension maximale du domaine et association à la porté large. (cf. supra §3.3).

Qui que ce soit délimite le domaine aux humains, c'est-à-dire un domaine d'individus appartenant à la même "sorte", d'extension très restreinte, et dont l'élargissement reste limité. En outre, il semble très facile en contexte, de délimiter l'ensemble des humains possibles concernés par l'énoncé. Dans (117) par exemple : il s'agit de la totalité des voyageurs, des passants, etc... selon le contexte de discours. En revanche, quoi que ce soit couvre un domaine beaucoup plus large, l'ensemble des entités possibles (sauf les humains), quelle que soit leur sorte, et dont l'élargissement semble sinon infini, du moins très étendu.

Qui que ce soit, d'extension plus restreinte, aura donc, comme les structures complètes à relative, mais à un degré moindre, une capacité d'interprétation comme universel à portée étroite qui fait en général défaut à quoi que ce soit quantificateur de domaine incomparablement plus étendu. ${ }^{19}$

Ce contraste conduit à amender légèrement la théorie, en limitant l'obligation absolue de portée large au seul quoi que ce soit, mais en réalité, il la confirme, car il repose en réalité sur la corrélation domaine maximal/portée large.

\subsection{Les rapports entre notre analyse et celle de Vlachou (2007).}

Le présent travail est inspiré par les propositions de Vlachou (2007) et en constitue pour nous un prolongement.

Vlachou catégorise quoi que ce soit comme un "concessive FCI", ce qui est parfaitement en accord avec notre proposition que la sémantique de l'expression a pour source une disjonction

19 où que ce soit, semble, comme qui que ce soit admettre plus facilement une interprétation universelle à portée étroite. Je n'accepte pas de travailler où que ce soit s'interprète aisément comme : "Je n'accepte pas de travailler partout". Comme qui que ce soit cette forme réduit considérablement l'extension du domaine (à des lieux). 
dont la sémantique est de type "concessive conditional", et la tentative de dérivation compositionnelle de cette sémantique proposée ici.

Pour expliquer les distributions, Vlachou postule que l'élargissement implique que toutes les alternatives puissent être la valeur de la variable associée (op. cit., p. 173). Cette formulation est compatible avec le traitement comme universel de portée large que je propose, mais n'est pas tout à fait équivalente. Si la forme est indéfinie, ce sont des effets de contexte qui doivent expliquer les interprétations universelles. Si la forme est universelle, les interprétations universelles sont inhérentes, et l'analyse a seulement pour charge d'expliquer qu'en certains contextes elles soient bloquées.

J'ai expliqué les blocages en postulant une interprétation de quoi que ce soit comme universel à portée large et domaine maximal, en supposant que cette généralité extrême était incompatible avec des énoncés particuliers. Ainsi, "Quoi que ce soit m'est tombé sur la tête" est non acceptable, alors que "Tout m'est tombé sur la tête" est possible; la raison en est que le premier quantifie sur la totalité de tous les individus possibles, qui ne peut pas être l'argument d'un énoncé épisodique, alors que tout admet typiquement des restrictions contextuelles de son domaine. De même, le fait que quoi que ce soit ne soit acceptable que dans la portée d'un opérateur décroissant peut selon moi être expliqué sur les mêmes bases. Les opérateurs décroissants sont compatibles avec une quantification universelle à domaine maximal de leur argument, ce qui n'est pas le cas, en général des autres opérateurs.

Pour expliquer le fonctionnement en contexte décroissant, Vlachou se fonde sur l'idée que l'élargissement implique que toutes les alternatives doivent pouvoir être la valeur de la variable associée (op. cit., p. 173), ce qui selon elle est automatiquement garanti par le type d'inférence "descendante" imposé par les opérateurs décroisssants. ${ }^{20} \mathrm{~J}^{\prime}$ observe ici qu'en effet les opérateurs décroissants ont la propriété d'accepter une quantification universelle maximale de leur argument, ce qui est une formulation très proche, mais je ne propose pas d'explication de cette corrélation, qui resterait selon moi a expliciter.

Pour ce qui concerne la différence d'acceptabilité de quoi que ce soit avec les modalités pouvoir (acceptable) devoir (peu acceptable), Vlachou utilise la proposition d'Aloni (2002). Celle-ci fait de pouvoir un opérateur de quantification universelle et de devoir un opérateur existentiel, opérateurs qui quantifient sur les alternatives obtenues en faisant varier la valeur de la variable "indéfinie" introduite par quoi que ce soit. Grossièrement, l'idée est que "Il peut soulever un cheval", asserte que toutes les alternatives (il peut soulever le cheval a, le cheval b, etc.) sont compatibles avec ce que nous croyons vrai; en revanche "Il doit soulever un cheval" asserte que au moins une des alternatives est impliquée.

Si quoi que ce soit est indéfini, il doit être lié par le quantificateur modal : l'élargissement impliquant que toutes les alternatives, sans exception, doivent être des valeurs possibles, il sera grammatical dans le cas de pouvoir (quantificateur universel) ; Il ne le sera pas pour le quantificateur existentiel associé à devoir.

$20 \quad$ "Les opérateurs décroissants autorisent des inférences à partir d'un ensemble d'alternatives vers chacun de ses sous-ensembles. Par conséquent, toutes les alternatives de l'ensemble A' que le FCI d'élargissement introduit, peuvent être ses valeurs. En conséquence, la sémantique du FCI qu-que ce soit qui requiert que toutes les alternatives puissent être ses valeurs est satisfaite" (op. cit., p. 176). Le lien direct établi entre monotonie décroissante et élargissement n'est pas très clair pour moi. Contrairement à ce que proposent Kadmon et Landman, il ne repose pas sur la notion de renforcement, et à la différence d'une explication comme celle de Dayal, que j'emprunte ici, il n'utilise pas la notion de quantification sur tous les individus possibles. 
L'explication est particulièrement élégante et rend compte directement d'un contraste inattendu entre les modalités du possible et du nécessaire. Mais elle a aussi des inconvénients. Elle est tout d'abord très forte, on ne devrait avoir aucun cas de transgression; ensuite, elle suppose une analyse non-standard et non classique des modalités. On considère plutôt devoir comme un universel sur les mondes possibles, et pouvoir comme un existentiel. Cette hypothèse suppose crucialement une analyse de quoi que ce soit comme indéfini, et traite le quantificateur modal comme le quantificateur qui lie la variable.

Comment le contraste se présente-t-il dans le cadre de notre analyse de quoi que ce soit comme universel?

Supposons que quoi que ce soit soit toujours un universel à portée large quantifiant sur tous les individus possibles. Pouvoir peut être analysé, classiquement, comme un quantificateur existentiel sur les mondes possibles accessibles.

(119) Pierre peut battre qui que ce soit

$=$ Pour tout individu possible $\mathrm{x}$, Il existe un monde accessible dans lequel Pierre bat $\mathrm{x}$.

Pour la modalité devoir, les volitionnelles et les impératives, si on admet une analyse classique comme quantification universelle sur les mondes accessibles on obtient pour (120):

(120) ?Pierre doit battre qui que ce soit.

$=$ Pour tout individu possible $\mathrm{x}$, dans tout monde accessible recherché, Pierre bat $\mathrm{x}$.

La malformation pourrait tenir ici à ce qu'il est impossible qu'un argument aussi étendu (par maximalité) soit requis de vérifier une proposition particulière dans le monde. Ce serait la maximalité (la prise en compte de toutes les alternatives possibles) qui serait la cause de l'agrammaticalité. Il semble donc possible de proposer dans une approche "universelle" une explication rivale de la théorie d'Aloni, laquelle repose sur une analyse "indéfinie" de any.

\section{Conclusion.}

Nous proposons dans cet article une analyse unitaire de quoi que ce soit comme quantificateur universel particularisé par deux propriétés : il prend toujours portée maximale, et quantifie "sans exception" sur l'ensemble des objets possibles (maximalité). Ce quantificateur est donc imperméable aux effets de restriction contextuelle du domaine qui affectent typiquement les autres quantificateurs linguistiques.

Une dérivation compositionnelle de cette sémantique a été proposée à partir de la structure complète quoi que ce soit + relative, analysée comme disjonction généralisée à interprétation de type "concessive conditional", donc comme quantification universelle forte sur des alternatives. Cette approche permet de dériver de façon compositionnelle le phénomène d'élargissement, pièce cruciale de la théorie de Vlachou (2007) sur quoi que ce soit, de deux sources : 1) les disjonctions généralisées imposent une quantification universelle forte ("sans exception") sur chacune des alternatives; 2) l'absence de relative dans l'emploi absolu de quoi que ce soit impose de quantifier sur tous les individus possibles. Au cours de ce travail, nous avons utilisé le terme d'élargissement sans toujours distinguer clairement ces deux traits et pour identifier le résultat : une quantification universelle forte sur tous les individus possibles. Cependant, la présente étude conduit à bien distinguer les deux propriétés. La quantification forte (sans exception) est le fait des complétives disjonctives, et la maximalité est le fait des usages absolus de quoi que ce soit comme argument. En un sens par conséquent, notre approche de quoi que ce soit ne fait pas un usage crucial de la notion d'élargissement en tant que telle, et reste parfaitement neutre quant à la nécessité d'introduire cette notion comme 
catégorie explicative dans la grammaire. $^{21}$

La forme réduite quoi que ce soit s'interprète donc universellement, comme la structure complète, et ne se distingue que par deux propriétés spécifiques : l'exigence de portée maximale, et la maximalité du domaine d'interprétation.

Loin de s'opposer à l'approche antérieure de Vlachou (2007) sur la sémantique de quoi que ce soit, le présent travail en serait plutôt une version différente qui s'en écarte sur le présupposé initial : quoi que ce soit est vu par nous comme un universel, alors que Vlachou le traite comme un indéfini. ${ }^{22}$ Les raisons qui motivent ce choix d'analyser quoi que ce soit comme un quantificateur universel sont multiples :

1. l'intuition des locuteurs, qui le rapprochent le plus souvent de tout;

2. la dérivation compositionnelle de cette expression qui n'est pas un atome lexical, mais un fragment de construction dont l'interprétation universelle est dérivable au moyen de processus à l'œuvre ailleurs dans la grammaire du français;

3. les difficultés des analyses comme "indéfini", c'est-à-dire comme forme dépourvue de toute force quantificationelle, à expliquer l'émergence de l'universel. Kadmon \& Landman (1993) utilisent la notion d'interprétation générique, mais cela soulève de nombreuses difficultés. Vlachou (2007) n'utilise pas cette voie, mais inclut dans sa notion d'élargissement une contrainte que l'on peut voir comme une analyse universelle implicite.

Par ailleurs nous suivons ici exactement la même voie que Vlachou (2007) : nous essayons de justifier les cas d'agrammaticalité comme incompatibilité entre la sémantique de l'item (pour nous universel à portée large impliquant un domaine maximal) et celles du contexte où il se trouve.

\section{Bibliographie}

Aloni, Maria, 2002, "Free choice in modal contexts", /in/ Weisgerber M. (éd.), / Proceedings of Sinn and Bedeutung/ 7, 25-37.

Anscombre, Jean-Claude, 2006, "Tout, n'importe quel, chaque. Quelques remarques", /in/ Francis Corblin, Sylvie Ferrando et Lucien Kupferman (éd.)/Indéfini et Prédication/, Presses universitaires de Paris-Sorbonne, 217-233.

Corblin, Francis, 2002, /Représentation du discours et sémantique formelle/, PUF.

Dayal, Veneeta, 1995, "Licensing any in-non-negative/non-modal contexts", SALT 5,72-93. Dayal, Veneeta , 1998, "ANY as Inherently Modal", /Linguistics \& Philosophy/ 21, 433-476. Dayal, Veneeta, 2004, " The Universal Force of Free Choice Any", /Linguistic Variation Yearbook/ 4, 5-40.

Déprez, Viviane, Martineau France, 2006, "Pour une approche Micro-paramétrique de la Concordance Négative Francophone", /in/ Francis Corblin, Sylvie Ferrando et Lucien Kupferman (ed) / Indéfini et Prédication/, Presses universitaires de Paris-Sorbonne, 217-233. Eisner, Jason, 1995," ' $\forall$ '-less in wonderland? revisiting any", /In/ Fuller Janet, Han Ho,

21 Voir les réserves exprimées dans l'article de Jayez et Tovena (ce volume) quant à la valeur explicative de cette notion. Je remercie Lucia Tovena pour ses commentaires sur le statut de la notion d'élargissement dans la présente étude.

22 Sur les parentés et différences entre les analyses "universelles" et "indéfinies" de any, on pourra se reporter à Dayal (2004). 
Parkinson David (éd.) /Proceedings of ESCOL11/, Ithaca, NY: DMLLPublications.

Fauconnier, Gilles, 1979, "Implication Reversal in a Natural Language",/in/ Guentner F. , Schmidt ,S.J. (éd.), /Formal Semantics and Pragmatics for Natural Languages/, 289-301.

Fauconnier, Gilles, 1975, "Pragmatic scales and logical structures", /Linguistic Inquiry/, 6, 353-375.

Gaatone, David, 1971, /Etude descriptive du système de la négation en français contemporain/, Droz, Genève.

Giannakidou, Anastasia, 1997, /The landscape of polarity/, Dissertation, University of Groningen, The Netherlands.

Giannakidou, Anastasia, 2001, "The meaning of free choice", /Linguistics and Philosophy/, 24, 659-735.

Hadermann, Pascale, 2008, "De la concession au libre choix en passant par la polarité : le cas de où que $P$ et de n'importe où", /Langue française/ 158, 116-128.

Haspelmath, M., König, E., 1998, "Concessive Conditionals in the Languages of Europe", /in/ Van der Auwera (éd.), /Adverbial relations in the language of Europe/, Mouton de Gruyter, 563-640.

Haspelmath, Martin, 1997, /Indefinite Pronouns/, Oxford: Clarendon.

Heim, Irene, 1982, /The semantics of definite and indefinite noun phrases/, Doctoral

Dissertation, University of Massachussetts at Amherst, Published in 1989 by Garland, New York.

Hintikka, Jaako, 1980, "On the any-thesis and the methodology of linguistics", /Linguistics and Philosophy/, 31, 401-424.

Horn, Laurence, 1972, /On the Semantic Properties of Logical Operators in English/, UCLA Dissertation, Indiana Linguistic Club, 1976.

Horn, Laurence, 2000, "Any and (-)ever: Free choice and free relatives", /in/ Proceedings of the 15th Annual Conference of the Israeli Association for Theoretical Linguistics, 71-111. Horn, Laurence, 2005, "Airport'86 revisited : toward a unified indefinite any", /in/ Carlson, G.N., Pelletier, J. (éd.) /Reference and Quantification, The Parteee effect/ ,CSLI, 179-203. Jacobson, Pauline, 1995, "On the quantificational force of English free relatives", /in/ Bach , E., Jelinek, E., Kratzer, A., Partee, B. (éd.) /Quantification in natural language/, Kluwer Academic Publishers, 451-486.

Jayez, Jacques, Tovena, Lucia, 2005, "Free choiceness and non-individuation", /Linguistics and Philosophy/, 28, 1-71.

Jayez, Jacques, Tovena, Lucia, 2010, "Quatre problèmes pour le choix libre", Ce volume. Kadmon, Nirit, Landman, Fred, 1993, "Any", /Linguistics and Philosophy/, 16, 353-422. Krifka M., Pelletier F.J., Carlson G.N., , ter Meulen A. ,Chierchia G., G. Link G., 1995, "Genericity: an introduction," /in//The Generic Book/, U. of Chicago Press, 1-124.

Krifka, Manfred, 1995, "The semantics and pragmatics of weak and strong polarity in assertion", SALT IV, 195-219.

Krifka, Manfred, 1995, "The semantics and Pragmatics of Polarity Items", /Linguistic Analysis/, 25, 146-180.

Kroch, Antony, 1975, /The semantics of scope in English/, Doctoral Dissertation, MIT, Bloomington: Indiana University Linguistics Club.

Ladusaw, William,1979, /Polarity sensitivity as Inherent Scope Relations/, .U. of Texas Dissertation, Garland Publishing, 1980.

Larrivée, Pierre, 2007, "La scalarité d'indéfinis à sélection arbitraire", /Travaux de linguistique/, 54, 97-107.

Larrivée, Pierre, 2007, /Du tout au rien: libre-choix et polarité négative/, Bibliothèque de grammaire et de linguistique, Paris: Champion.

Lasnik, Howard, 1972, /Analyses of negation in English/, Doctoral Dissertation, 
Massachussetts Institute of Technology.

LeGrand, Jean Ehrenkranz, 1975, /Or and Any: The semantics and syntax of two logical operators/, Doctoral Dissertation, University of Chicago, Department of Linguistics.

Leuschner, T., 2006, /Hypotaxis as building-site : the emergence and grammaticalization of concessive conditionals in English, German and Dutch/, Lincom Europa.

Lewis, David, 1975, "Adverbs of quantification", /in/ Keenan E.L.,(éd.), /Formal Semantics of Natural Language/, Cambridge: Cambridge University Press.

Martin, Robert, 2002, /Comprendre la linguistique/, Quadrige, PUF.

Muller, Claude, 2006, "Polarité négative et free-choice dans les indéfinis de type "que ce soit" et "n'importe"", /Langages/, 162, 7-31.

Muller, Claude, 2007, "Les indéfinis free-choice confrontés aux explications scalaires",

/Travaux de linguistique/, 54, 83-96.

Muller, Claude, 2008, "Valeurs communes et valeurs particulières des formes QU- en

français", /Langue française/, 158, 13-28.

Pierrard, Michel, 2009, "Comment et les expressions indéfinies en n'importe et que ce soit", /Travaux de linguistique/, 58, 127 à 148.

Quine, W.V.O, 1960, /Word and object/ Cambridge, Mass: MIT press.

Reed, Paul, 2000, "'Any" and its French equivalents", /French Language Studies/, 10, 101116.

Reichenbach, H., 1947, /Elements of symbolic logic/, New York: The Free Press.

Szabolcsi, A., 2004, "Positive polarity -- negative polarity", /Natural Language and Linguistic Theory/, 22, 409-452.

Tovena, Lucia, Déprez, Viviane, Jayez, Jacques, 2004, "Polarity Sensitive Items" /in/ Corblin, F., De Swart, H., (éd.), /Handbook of French Semantics/, CSLI Publications.

Vendler, Zeno, 1967, /Linguistics in philosophy/, Ithaca: Cornell University Press.

Vlachou, Evangelia, 2007, /Free Choice in and out of Context: semantics and Distribution of French, Greek and English Free Choice Items/, Utrecht and Paris-Sorbonne dissertation, Utrecht Institute of Linguistics OTS.

Vlachou, Evangelia, 2009, "Indefinite free choice items in necessity modal contexts", /in/ Harpert C. et al. (éd) /Proceedings of the 2007 Workshop in Greek Syntax and Semantics at MIT/, MIT working papers in linguistics 57.

Zwarts, Frans, 1995, "Nonveridical contexts", /Linguistic Inquiry/, 25, 286-312. 